\title{
GENERALIZED JACOBI POLYNOMIALS
}

D. N. SEN AND V. RANGACHARIAR

1. Introduction. The differential equation

$$
\left(\alpha x^{2}+\beta x+\gamma\right) \frac{d^{2} y}{d x^{2}}-\left(x+a_{1}\right) \frac{d y}{d x}+\{n-n(n-1) \alpha\} y=0,
$$

where $n$ is a positive integer, has polynomial solutions $y_{n}$ of degree $n$. Some properties of these polynomials have been obtained by Brenke* and by Lawton. $\uparrow$ The object of this paper is to derive fresh properties and in particular to study the zeros of these polynomials. Brenke proved that

$$
h_{n} y_{n}=\frac{1}{\rho} D^{n}\left\{\rho P^{n}\right\}
$$

where

$$
\begin{aligned}
P & \equiv \alpha x^{2}+\beta x+\gamma \equiv-\alpha(x-a)(b-x), \quad(a<b), \\
\rho & \equiv-\frac{1}{\alpha}(x-a)^{A-1}(b-x)^{B-1} \\
A & =\frac{a+a_{1}}{\alpha(b-a)}, \quad B=\frac{b+a_{1}}{-\alpha(b-a)},
\end{aligned}
$$

and $h_{n}$ is the coefficient of $x^{n}$ in the right-hand side. It has also been proved by him that if $A$ and $B$ are positive, the following recurrence formula holds good.

$$
y_{n}=\left(a_{n}+x\right) y_{n-1}-b_{n} y_{n-2},
$$

where

$$
\begin{aligned}
& b_{n}=\frac{c_{n-2}^{2}}{c_{n-1}^{2}} \text { and } \frac{1}{c_{n}^{2}}=\int_{a}^{b} \rho y_{n}^{2} d x, \\
& a_{n}=-c_{n-1}^{2} k_{n-1}, \quad \text { where } \quad k_{n}=\int_{a}^{b} x \rho y_{n}^{2} d x .
\end{aligned}
$$

* This Bulletin, vol. 36 (1930).

$\dagger$ This Bulletin, vol. 38 (1932). 
The values of $A$ and $B$ have been assumed to be positive for the existence of the integral $\int_{a}^{b} \rho d x$.

2. Values of $y_{n}$ at $a$ and $b$.

$$
\begin{aligned}
h_{n} y_{n}= & \frac{1}{\rho} D^{n}\left(\rho P^{n}\right)=\frac{(-\alpha)^{n-1}}{\rho} D^{n}\left\{(x-a)^{n+A-1}(b-x)^{n+B-1}\right\} \\
= & (-\alpha)^{n}(x-a)^{1-A}(b-x)^{1-B} D^{n}\left\{(x-a)^{n+A-1}(b-x)^{n+B-1}\right\} \\
= & (-\alpha)^{n}(x-a)^{1-A}(b-x)^{1-B} \\
& \cdot\left[\sum_{r=0}^{+n}(-1)^{r} C_{n, r} S_{r}(x-a)^{r+A-1}(b-x)^{n-r+B-1}\right],
\end{aligned}
$$

where

$$
\begin{aligned}
S_{r} & =(n+A-1)(n+A-2) \cdots(A+r) \\
& \times(n+B-1)(n+B-2) \cdots(n+B-r) .
\end{aligned}
$$

Now $h_{n}=\alpha^{n}(2 n+A+B-2)(2 n+A+B-3) \cdots(n+A+B-1)$. Hence

$$
\begin{aligned}
& y_{n}(a)=(-1)^{n} \frac{(n+A-1)(n+A-2) \cdots A(b-a)^{n}}{(2 n+A+B-2)(2 n+A+B-3) \cdots(n+A+B-1)}, \\
& y_{n}(b)=\frac{(n+B-1)(n+B-2) \cdots B(b-a)^{n}}{(2 n+A+B-2)(2 n+A+B-3) \cdots(n+A+B-1)} .
\end{aligned}
$$

3. Value of $b_{n}$ in formula (A).

$$
\begin{aligned}
\int_{a}^{b} \rho y_{k}^{2} d x & =\frac{1}{h_{k}^{2}} \int_{a}^{b} D^{k}\left(\rho P^{k}\right)\left\{\frac{1}{\rho} D^{k}\left(\rho P^{k}\right)\right\} d x \\
& =\frac{(-1)^{k}}{h_{k}^{2}} \int_{a}^{b} \rho P^{k} D^{k}\left\{\frac{1}{\rho} D^{k}\left(\rho P^{k}\right)\right\} d x \\
& =\frac{(-1)^{k} k !}{h_{k}} \int_{a}^{b} \rho P^{k} d x \\
& =\frac{(-1)^{k} k !}{h_{k}}(-\alpha)^{k-1} \int_{a}^{b}(x-a)^{k+A-1}(b-x)^{k+B-1} d x \\
& =\frac{(-1)^{k} k !}{h_{k}}(-\alpha)^{k-1} \frac{\Gamma(k+A) \Gamma(k+B)}{\Gamma(2 k+A+B)}(b-a)^{2 k+A+B-1} .
\end{aligned}
$$


Hence

$$
\begin{aligned}
b_{n} & =\frac{\int_{a}^{b} \rho y_{n-1}^{2} d x}{\int_{a}^{b} \rho y_{n-2}^{2} d x} \\
& =\alpha \frac{h_{n-2}}{h_{n-1}}(n-1) \frac{(n+A-2)(n+B-2)}{(2 n+A+B-3)(2 n+A+B-4)}(b-a)^{2} \\
& =(n-1) \frac{(n+A-2)(n+B-2)(n+A+B-3)}{(2 n+A+B-3)(2 n+A+B-4)^{2}(2 n+A+B-5)}(b-a)^{2} .
\end{aligned}
$$

4. Relation among $y_{n}^{\prime}, y_{n}, y_{n+1}$. From $\$ 2$

$$
h_{n} y_{n}=(-\alpha)^{n} \sum_{r=0}^{r=n}(-1)^{r} S_{r} C_{n, r}(x-a)^{r}(b-x)^{n-r} .
$$

\section{Hence}

$$
\begin{aligned}
h_{n} y_{n}^{\prime}=(-\alpha)^{n} \sum_{0}^{n}(-1)^{r} S_{r} C_{n, r} & \left\{r(x-a)^{r-1}(b-x)^{n-r}\right. \\
& \left.-(n-r)(x-a)^{r}(b-x)^{n-r-1}\right\} .
\end{aligned}
$$

The coefficient

$$
(x-a)^{r}(b-x)^{n-r-1}
$$

on the right-hand side under summation is

$$
\begin{aligned}
& (-1)^{r+1} C_{n, r+1}(r+1) S_{r+1}+(-1)^{r+1}(n-r) C_{n, r} S_{r} \\
& =(-1)^{r+1} n C_{n-1, r}\left\{S_{r}+S_{r+1}\right\} \\
& =(-1)^{r+1} n C_{n-1, r}\{(A+r)+(n+B-r-1)\}(n+A-1) \\
& \quad \cdots(A+r+1) \times(n+B-1) \cdots(n+B-r) \\
& =(-1)^{r+1} n C_{n-1, r}(n+A+B-1)(n+A-1) \\
& \quad \cdots(A+r+1) \times(n+B-1) \cdots(n+B-r) .
\end{aligned}
$$

Therefore

$$
\begin{aligned}
h_{n} y_{n}^{\prime} & =-(-\alpha)^{n} n(n+A+B-1) \\
& \times \sum_{r=0}^{n-1}\left\{(-1)^{r} C_{n-1, r}(n+A-1) \cdots(A+r+1)\right. \\
& \left.\times(n+B-1) \cdots(n+B-r) \times(x-a)^{r}(b-x)^{n-r-1}\right\} .
\end{aligned}
$$

Now 


$$
\begin{aligned}
& \{(n+A)(b-x)-(n+B)(x-a)\} h_{n} y_{n} \\
& =\{(n+A)(b-x)-(n+B)(x-a)\}(-\alpha)^{n} \\
& \cdot \sum_{r=0}^{n}(-1)^{r} S_{r} C_{n, r}(x-a)^{r}(b-x)^{n-r} .
\end{aligned}
$$

The coefficient of $(x-a)^{r+1}(b-x)^{n-r}$ on the right-hand side is

$$
\begin{aligned}
& (-\alpha)^{n}(-1)^{r+1}\left\{(n+B) C_{n, r} S_{r}+(n+A) C_{n, r+1} S_{r+1}\right\} \\
& =(-\alpha)^{n}(-1)^{r+1}\left\{(n+B)(A+r) C_{n, r}\right. \\
& \left.\quad+(n+A)(n+B-r-1) C_{n, r+1}\right\} \\
& \quad \times(n+A-1) \cdots(A+r+1) \\
& \quad \times(n+B-1) \cdots(n+B-r) .
\end{aligned}
$$

The expression within the braces is

$$
\begin{aligned}
& (n+B)(n+A-\overline{n-r}) C_{n, r}+(n+A)(n+B-\overline{r+1}) C_{n, r+1} \\
& \quad=(n+A)(n+B)\left(C_{n, r}+C_{n, r+1}\right)-n C_{n-1, r}(2 n+A+B) \\
& \quad=(n+A)(n+B) C_{n+1, r+1}-n C_{n-1, r}(2 n+A+B) .
\end{aligned}
$$

\section{Hence}

$$
\begin{aligned}
\{(n+A)(b-x)-(n+B)(x-a)\} h_{n} y_{n} \\
=(-\alpha)^{n} \sum_{0}^{n+1}(-1)^{r} C_{n+1, r}(n+A)(n+A-1) \cdots(A+r) \\
\times(n+B) \cdots(n+B-r+1) \times(x-a)^{r}(b-x)^{n-r+1} \\
\quad+(-\alpha)^{n} n(2 n+A+B) \\
\quad \cdot \sum_{0}^{n-1}(-1)^{r} C_{n-1, r}(n+A-1) \cdots(A+r+1) \\
\times(n+B-1) \cdots(n+B-r)(x-a)^{r+1}(b-x)^{n-r} \\
=\frac{h_{n+1}}{-\alpha} y_{n+1}+(-\alpha)^{n} n(2 n+A+B) \\
\quad \cdot \sum_{0}^{n-1}(-1)^{r} C_{n-1, r}(n+A-1) \cdots(A+r+1) \\
\times(n+B-1) \cdots(n+B-r)(x-a)^{r+1}(b-x)^{n-r} .
\end{aligned}
$$

Also 


$$
\begin{gathered}
(x-a)(b-x) h_{n} y_{n}^{\prime}=-n(n+A+B-1)(-\alpha)^{n} \\
\cdot \sum_{0}^{n-1}(-1)^{r} C_{n-1, r}(n+A-1) \cdots(A+r+1) \\
\times(n+B-1) \cdots(n+B-r)(x-a)^{r+1}(b-x)^{n-r} .
\end{gathered}
$$

Hence

$$
\begin{aligned}
\{(n+A)(b & -x)-(n+B)(x-a)\} h_{n} y_{n} \\
& =\frac{h_{n+1}}{-\alpha} y_{n+1}-\frac{2 n+A+B}{n+A+B-1}(x-a)(b-x) h_{n} y_{n}{ }^{\prime} .
\end{aligned}
$$

Therefore

$$
\begin{aligned}
& (x-a)(b-x) y_{n}^{\prime}=-(2 n+A+B-1) y_{n+1} \\
& \quad+\frac{n+A+B-1}{2 n+A+B}\{(n+B)(x-a)-(n+A)(b-x)\} y_{n} .
\end{aligned}
$$

5. Results Valid for $A$ and $B$ Negative. If $A$ and $B$ be assumed to be negative, the results of $\S \S 2$ and 4 obviously continue to hold good. The recurrence formula (A), being an identity and $y_{n}^{\prime \prime}$ 's being polynomials, will still be true and the form for $b_{n}$ obtained in $\$ 3$ will also hold good.

6. Roots of $y_{n}=0$. Lawton and Fujiwara proved that if $A$ and $B$ be negative or zero, and $p, q$ positive integers such that $0<A+p \leqq 1,0 \leqq B+q \leqq 1$, the number of roots of $y_{n}=0$ inside $(a, b)$ is $n-p-q,(n \geqq p+q+1)$. We shall derive the result by arguments based on methods of Sturm's theorem in the theory of equations and get fresh results by this consideration. The set of functions $y_{n}, y_{n-1}, \cdots, y_{p+q}$ is taken for this purpose, it being proved in the next section that $y_{p+q}=0$ has no roots inside $(a, b)$.

7. No roots of $y_{p+q}=0$ inside $(a, b)$. When $x$ lies between $a$ and $b,(x-a)$ and $(b-x)$ are both positive. The coefficient of $(x-a)^{r}(b-x)^{n-r}$ on the right-hand side of $h_{n} y_{n}$ in $\$ 2$ is $(-\alpha)^{n} C_{n, r}(-1)^{r} S_{r}$. When $n=p+q$, this coefficient becomes

$$
\begin{aligned}
(-\alpha)^{p+q} C_{p+q, r}(-1)^{r}(p+q+A-1) & \cdots(A+r) \\
\times(p+q+B-1) & \cdots \times(p+q+B-r) .
\end{aligned}
$$

If $r=p-s$, where $s$ is positive or zero, 


$$
(p+q+A-1) \cdots(p+A-1) \cdots(A+p-s)
$$

has $s$ negative factors and in $(p+q+B-1) \cdots(q+B+s)$ all the factors are positive. Hence in this case the sign of $(-1)^{r} S_{r}$ is that of $(-1)^{p}$. If, however, $r=p+t$, where $t$ is positive, all the factors in $(p+q+A-1) \cdots(A+p+t)$ are positive and in $(p+q+B-1) \cdots(q+B-1) \cdots(q+B-t)$ there are $t$ negative factors. Hence again the sign of $(-1)^{r} S_{r}$ is that of $(-1)^{p}$. It follows that $h_{p+q} y_{p+q}$ cannot vanish within $(a, b)$ and has the same sign as that of $(-\alpha)^{p+q}(-1)^{p}$. Now

$$
\begin{aligned}
h_{p+q}=(+\alpha)^{p+q}(2 p+2 q+A+B-2) \\
\cdots(p+q+A+B)(p+q+A+B-1) .
\end{aligned}
$$

Hence the sign of $y_{p+q}$ is that of $(-1)^{q}(p+q+A+B-1)$, that is, the sign of $y_{p+q}$ within $(a, b)$ is that of $(-1)^{q}$ or $(-1)^{q-1}$ according as the sum of the fractional parts of $-A$ and $-B$ is $<1$ or $>1$. The case in which the sum $=1$ follows by continuity. ${ }^{*}$

8. Changes in Sign. In passing through a root $\theta$ of $y_{n}=0$ a change of sign will be lost or gained between $y_{n}$ and $y_{n-1}$ according as $y_{n}^{\prime}(\theta)$ and $y_{n-1}(\theta)$ have like or unlike signs, that is, according as $b_{n+1}$ is positive or negative ( $\$ 4$ and $(\mathrm{A})$ ). By formula (A) when $y_{k-1}=0, y_{k} / y_{k-2}$ will have sign opposite to that of $b_{k}$. If $b_{k}$ be positive, no change of sign will be lost or gained in passing through a root $\delta$ of $y_{k-1}=0$; if on the other hand $b_{k}$ be negative, two changes of sign will be lost or gained according as $y_{k-1}^{\prime}(\delta)$ and $y_{k-2}(\delta)$ are of the same or opposite sign. The relation of $\S 4$ enables us to deal with the different possibilities and an examination of the changes of sign lost enables us to determine the number of roots of $y_{n}=0$ within $(a, b)$.

9. Sign of $b_{n}$ for Different Values of $n$. For studying the zeros of $y_{n},(n \geqq p+q)$, it is enough to consider the sign of $b_{n}$ for $n \geqq p+q+2$. All the factors in $b_{n}$ except $n+A+B-3$ are evidently positive. This factor is also positive for $n \geqq p+q+2$ if the sum of the fractional parts of $-A$ and $-B$ be less than unity, but if this sum exceeds unity, then $b_{n}$ is negative for $n=p+q+2$, and positive for subsequent values of $n$.

* This re-statement is made to guard against the possibility that we may have $h_{p+q}=0$ in the fraction $(-\alpha)^{p+q}(-1)^{p} / h_{p+q}$. 
10. Sum of Fractional Parts $<1$. Thus if the sum of the fractional parts be $<1$, a change of sign will be lost in passing through a root of $y_{n}=0$ and no change of sign will be lost or gained for a root of any of the intermediate functions. Also from $\$ 2$, it is evident that at $b$ the functions are of the same sign and at $a$ consecutive ones are of opposite signs. Hence the number of roots of $y_{n}=0$ between $a$ and $b$ will be $n-p-q,(n \geqq p+q)$.

11. Sum of Fractional Parts $>1$. If, on the other hand, the sum of the fractional parts $>1$, at $x=b$ the signs of $y_{p+q}$ and $y_{p+q+1}$ are opposite and there will be continuation of sign for the subsequent functions; whereas at $a, y_{p+q}$ and $y_{p+q+1}$ will be of the same sign and then there will be variation of sign at each stage subsequently. Hence the number of changes of sign lost will be $n-p-q-2$. The equation $y_{p+q+1}=0$ will evidently have only one root in $(a, b)$. In passing through this root there will be two gains as $b_{p+q+2}$ is negative in this case. In passing through a root of $y_{n}=0,(n \geqq p+q+2)$, there will be a loss of sign between $y_{n}$ and $y_{n-1}$ and there will be no loss or gain for a root of any $y_{k}=0,(p+q+2 \leqq k \leqq n-1)$. Hence the number of roots of $y_{n}=0$ will exceed by 2 the number of changes of sign lost, that is, it will be $n-p-q,(n \geqq p+q)$. The result of Lawton is extended by our method to the case $n=p+q$.

12. Application of Sturm's Method. Sturm's method may be applied for the ranges $(-\infty, a)$ and $(b, \infty)$ as well. In particular we shall show that the number of roots of the equation $y_{n^{\prime \prime}}=0$ and $y_{n^{\prime}}=0$, where $n^{\prime \prime}>n^{\prime} \geqq[-(A+B)+3]=n_{0}$, is the same in either of these ranges, $[x]$ denoting the integral part of $x$. For studying such a range it is clear that we cannot stop at $y_{p+q}$, but we have to take the whole set of functions down to $y_{0}$. Let us take the sets $y_{0}, y_{1}, \cdots, y_{n^{\prime}}$ and $y_{0}, y_{1}, \cdots, y_{n^{\prime \prime}}$. In the first instance, the number of changes of sign in passing through $(-\infty, a)$ will be the same for the two sets. Also $b_{n+1}$ is positive for $n \geqq n_{0}$. Hence in the second set no change is contributed for passing through a root of $y_{n^{\prime}}=0, y_{n^{\prime}+1}=0, \cdots, y_{n^{\prime \prime}-1}=0$, which come as intermediate functions. Again the nature of the change for a root of the last function in the two cases will be the same, for $y_{n^{\prime}}^{\prime}\left(\theta^{\prime}\right) / y_{n^{\prime}-1}\left(\theta^{\prime}\right)$ and $y_{n^{\prime \prime}}^{\prime}\left(\theta^{\prime \prime}\right) / y_{n^{\prime \prime}-1}\left(\theta^{\prime \prime}\right)$ are of the same sign $\left[y_{n}^{\prime}(\theta) / y_{n^{\prime}-1}(\theta)\right.$ is negative in $(-\infty, a)$ for $\left.n \geqq n_{0}\right]$. Now in the first set the number of losses is due to (1) the roots of some inter- 
mediate functions and (2) the roots of $y_{n^{\prime}}=0$. In the second set the contribution due to (1) persists. By the introduction of the additional functions in the second set, the change contributed by a root of $y_{n^{\prime}}=0$ in the first set is transferred to a root of $y_{n^{\prime \prime}}=0$ in the second set. Also no change arises for a root of $y_{n^{\prime}}=0$, $y_{n^{\prime}+1}=0, \cdots, y_{n^{\prime \prime}-1}=0$. Since the total number of losses is the same in the two cases, the conclusion will be that the number of roots for $y_{n^{\prime}}=0$ and $y_{n^{\prime \prime}}=0$ in the range $(-\infty, a)$ will be the same; similarly for the range $(b, \infty)$. Also in $(a, b)$ the numbers of roots of $y_{n^{\prime}}=0$ and $y_{n^{\prime \prime}}=0$ are $n^{\prime}-p-q$ and $n^{\prime \prime}-p-q$. Hence we conclude that the number of imaginary roots of $y_{n^{\prime}}=0$ and $y_{n^{\prime \prime}}=0$ will be the same for $n^{\prime \prime}>n^{\prime} \geqq n_{0}$.

\section{SCIEnce College,}

PatNa, India

\section{NOTE ON THE EXISTENCE OF AN $n$ TH DERIVATIVE DEFINED BY MEANS OF A SINGLE LIMIT \\ BY NORMAN MILLER}

The $n$th derivative of a function $f(x)$ may be defined without the use of derivatives of lower order by means of the limit of a certain quotient. Conditions necessary and sufficient for the existence and continuity of $f^{(n)}(x)$ at a point $x=a$ and also for the mere existence of $f^{(n)}(a)$ have been recently given by Franklin.* The purpose of the present note is to state necessary and sufficient conditions of a somewhat more general form with proofs which use only Rolle's theorem and elementary properties of determinants.

Let $f_{i}(x)$ and $\phi_{i}(x),(i=1,2, \cdots, n+1)$, be functions possessing derivatives of the $n$th order, continuous in an interval $I$. Let $x_{1}, x_{2}, \cdots, x_{n+1}$ be points of $I$ which close down in an arbitrary manner on a point $a$, in the sense that

$$
\left|x_{j}-a\right|<\epsilon_{k}, \quad \lim _{k \rightarrow \infty} \epsilon_{k}=0 .
$$

We shall use the notation

\footnotetext{
* This Bulletin, vol. 41 (1935), p. 573.
} 